\title{
Indicadores de variabilidade glicêmica e mortalidade em pacientes críticos com terapia nutricional
}

\author{
Indicators of glycemic variability and mortality in critically ill patients with nutritional therapy
}

\section{DOI: 10.37111/braspeni.2019344008}

Josiane Monteiro Almeida

Lucas Lima de Carvalho²

\section{Unitermos:}

Terapia Nutricional. Unidade de Terapia Intensiva. Glicemia. Mortalidade. Hipoglicemia. Hiperglicemia.

\section{Keywords:}

Nutrition Therapy. Intensive Care Units. Blood Glucose. Mortality. Hypoglycemia. Hyperglycemia.

\section{Endereço para correspondência}

Josiane Monteiro Almeida

Alameda Oscar Niemeyer, 499 - Vila da Serra - Nova

Lima, MG, Brasil - CEP: 34000-000

E-mail: josimonteiroalmeida@hotmail.com

\section{Submissão}

4 de junho de 2019

Aceito para publicação

11 de setembro de 2019

\section{RESUMO}

Introdução: A variabilidade glicêmica (VG) tem sido associada à mortalidade em pacientes críticos. Vários métodos vêm sendo utilizados para medí-la, no entanto, nenhuma diretriz até o momento propôs um padrão-ouro. O objetivo deste estudo foi associar diferentes métodos de medida de VG com a mortalidade em pacientes de uma unidade de terapia intensiva (UTI), em vigência de terapia nutricional (TN). Método: Estudo longitudinal retrospectivo realizado entre abril e setembro de 2018, em que foram arrolados 82 pacientes em terapia intensiva, com mais de 48 horas de internação e em uso de TN. Foram coletados dados demográficos, clínicos e todos os valores de glicemia aferidos durante a internação. A VG foi avaliada por meio de três indicadores: desvio padrão (DP), coeficiente de variação (CV) e média glicêmica (MGL). Na análise estatística, foram utilizados os testes Whitney e Qui-quadrado de Pearson, regressão logística univariada e múltiplas, e a razão de chances (odds ratio). As análises foram processadas e avaliadas no software Statistical Data Analysis versão 12.0. Resultados: Foram aferidas 11.676 glicemias. A maior parte da amostra recebeu dieta enteral. A mortalidade foi de $42,7 \%$. Um total de $36,58 \%$ dos pacientes tinham diagnóstico prévio de diabetes mellitus (DM) e esses apresentaram valores maiores para todos os parâmetros avaliados de VG em relação aos não-diabéticos. Os valores de CV, DP e MGL foram maiores entre os pacientes que foram a óbito. A análise de regressão logística univariada demonstrou que as variáveis CV, DP e MGL tiveram associação significativa com a mortalidade. Mas, a partir do modelo de regressão logística multivariada, apenas o DP manteve relação com a mortalidade. Conclusão: O DP é um indicador de VG e está associado de modo independente com aumento de mortalidade em uma coorte de pacientes heterogêneos em uso de TN na UTI.

\section{ABSTRACT}

Introduction: Glycemic variability (GV) has been associated with mortality in critically ill patients. Several methods have been used to measure it, but no guideline has so far proposed a gold standard. The aim of this study was to associate different GV measurement methods with mortality in patients in an intensive care unit (ICU) under nutrition therapy (NT). Methods: Retrospective longitudinal study conducted between April and September 2018, in which 82 patients were enrolled in intensive care, with more than 48 hours of hospitalization and using NT. Demographic data, clinical data and all blood glucose values measured during hospitalization were collected. The GV was evaluated through three indicators: standard deviation (SD), coefficient of variation (CV) and glycemic mean (MGL). Statistical analysis was performed using Whitney and Pearson Chi-square tests, univariate and multiple logistic regression, and odds ratio. The analyzes were processed and evaluated using the Statistical Data Analysis software version 12.0. Results: 11,676 blood glucose levels were measured. Most of the sample received enteral diet. Mortality was $42.7 \%$. A total of $36.58 \%$ of the patients had a previous diagnosis of diabetes mellitus (DM) and these presented higher values for all evaluated parameters of VG compared to non-diabetics. CV, SD and MGL values were higher among the patients who died. Univariate logistic regression analysis showed that the variables CV, SD and MGL were significantly associated with mortality. However, based on the multivariate logistic regression model, only PD maintained a relationship with mortality. Conclusion: The SD is an indicator of GV is independently associated with increased mortality in a cohort of heterogeneous patients on TN in the ICU.

1. Especialista em Terapia Nutricional e Nutrição Clínica pelo GANEP Educação, São Paulo, SP, Brasil; membro da Equipe Multiprofissional de Terapia Nutricional do Hospital Vila da Serra, Nova Lima, MG, Brasil.

2. Especialista em Terapia Nutricional e Nutrição Clínica pelo GANEP Educação, São Paulo, SP, Brasil; membro da Equipe Multiprofissional de Terapia Nutricional do Hospital Vila da Serra, Nova Lima, MG, Brasil; Especialista em Medicina Intensiva pela Associação de Medicina Intensiva Brasileira (AMIB), São Paulo, SP, Brasil; Especialista em Nutrição Parenteral e Enteral pela Sociedade Brasileira de Nutrição Parenteral/BRASPEN São Paulo, SP, Brasil; Pós-graduado Latu Sensu em Nutrição Clínica pela Associação Brasileira de Nutrologia (ABRAN), Catanduva, SP, Brasil. 


\section{INTRODUÇÃO}

O controle glicêmico na unidade de terapia intensiva (UTI) tem sido um tema de grande interesse desde a publicação do estudo de Van den Berghe et al. ${ }^{1}$, em 2001, em que os autores correlacionaram a hiperglicemia no paciente crítico com aumento de complicações e mortalidade, e que a insulinoterapia intensiva poderia reduzir tais desfechos. Depois deste trial, surgiram vários outros fazendo a mesma correlação, definindo assim a importância de um controle glicêmico adequado nas UTIs. É notório que a hiperglicemia e o aumento da resistência insulínica no paciente gravemente enfermo são prevalentes e fazem parte de uma complexa resposta endócrino-metabólica do organismo a uma agressão, independente da presença ou não de diabetes mellitus (DM). E a maioria dos estudos que tentou definir uma meta glicêmica usou os conceitos "hiperglicemia" e "hipoglicemia" como preditores de complicações ${ }^{2-5}$.

De alguns anos para cá, surge o interesse em outro parâmetro, tão importante quanto os valores glicêmicos acima ou abaixo do normal: a variabilidade glicêmica (VG), ou seja, as flutuações agudas dos níveis de glicemia ao redor de um valor médio ${ }^{6-8}$.

Vários estudos retrospectivos demonstraram que a VG pode estar associada à mortalidade em pacientes críticos. Egi et al. ${ }^{9}$, estudando uma coorte multicêntrica $(n=7049)$, concluíram que a VG é um biomarcador independente de mortalidade. Após análise retrospectiva de 5728 pacientes de uma UTI cirúrgica, Hermanides et al. ${ }^{10}$ mostraram que a VG está firmemente associada à morte hospitalar. Resultados semelhantes foram encontrados em uma coorte de pacientes com sepse e em uma outra UTI cirúrgica ${ }^{11,12}$.

A VG pode ser representada por desvio padrão (DP), média glicêmica (MGL) ou coeficiente de variação glicêmica (CV). O DP é o parâmetro mais comumente usado, embora nenhum padrão-ouro para medir a VG tenha sido estabelecido ainda. Dentre os parâmetros existentes, o DP parece ser o melhor preditor de mortalidade ${ }^{13}$.

Em estudo realizado por Krinsley ${ }^{14}$, em uma população de UTI, o DP como marcador de VG foi um preditor de mortalidade dentro de diferentes faixas de glicemia e um preditor mais forte do que a própria glicemia média.

A VG pode ser afetada por fatores exogénos, como a infusão de certos medicamentos, a qualidade do monitoramento da glicemia, o cálculo adequado da taxa de insulina e a infusão da dieta enteral e/ou parenteral ${ }^{11}$. A terapia nutricional (TN), apesar de melhorar o status nutricional do paciente, pode contribuir para os distúrbios na glicemia aliados a alterações hormonais e inflamatórias ${ }^{15}$.

O intuito deste estudo é investigar se existe associação de forma independente entre a VG, em seus três domínios (DP, MGL e CV), com a mortalidade em pacientes críticos em uso de TN.

\section{MÉTODO}

Trata-se de um estudo longitudinal retrospectivo, realizado entre abril e setembro de 2018, em que foram avaliados pacientes de uma UTI de um Hospital em Nova Lima-MG.

Foram incluídos no estudo todos os pacientes com idade mínima de 20 anos, de ambos os sexos, que permaneceram pelo menos 48 horas internados na UTI e que receberam TN enteral e/ou parenteral durante a internação. Foram excluídos pacientes que não se enquadraram nos critérios pré-determinados e aqueles que se recusaram a participar.

Nos prontuários, foram coletados todos os valores de glicemia aferidos durante a internação (conforme protocolo institucional), diagnóstico, idade, sexo, história pregressa, peso, altura, tipo de TN utilizada e evolução clínica até seu desfecho.

As variáveis do estudo contemplaram parâmetros demográficos, hospitalares, características de saúde e parâmetros de VG dos pacientes (DP, MGL, CV). As análises foram estratificadas entre grupos de diabéticos e não-diabéticos, óbitos e sobreviventes da UTI, óbitos e não-óbitos de pacientes diabéticos. Para representação dos resultados, as variáveis com distribuição não-paramétrica foram apresentadas em mediana e intervalo interquartílico (p25-p75) e número absoluto e proporções correspondentes. Para a análise estatística entre os grupos foram utilizados os testes de Mann Whitney e Qui-quadrado de Pearson, para identificar diferenças ao nível de significância de 95\%. Para verificar a distribuição das variáveis, o teste de Shapiro Wilk foi utilizado. A associação entre mortalidade e características demográficas, hospitalares e parâmetros de VG foi testada primeiramente por análise bivariada a partir de modelos de regressão logística simples, tendo como variável dependente a mortalidade na UTI. Após análise univariada, as variáveis explicativas que obtiveram valor $p$ inferior a $20 \%(p<0,20)$ foram inseridas pelo método de backward no modelo multivariado de regressão logística múltipla, sendo que aquelas com menor significância foram retiradas uma a uma do modelo. O procedimento foi repetido até que todas as variáveis possuíssem significância estatística $(p<0,05)$. Para ajuste do modelo final, o teste de Hosmer e Lemeshow foi utilizado. A razão de chances (odds ratio) com intervalo de confiança de $95 \%$ foi utilizada como medida de efeito.

Os dados foram processados no programa Epi Info versão 7 e analisados no software Statistical Data Analysis versão 12.0.

Os participantes do presente estudo foram recrutados por meio de convite pessoal ou ao familiar responsável, após preenchidos os critérios de inclusão. Ao concordarem em participar de maneira voluntária, foi assinado o Termo de Consentimento Livre e Esclarecido. Os pacientes que foram a óbito antes do início da coleta de dados foram incluídos na pesquisa com base na resolução $N^{\circ} 466$, de 12 de dezembro 
de 2012. O presente estudo foi aprovado pelo comitê de ética em pesquisa do Centro Universitário UNA - MG (parecer $\left.n^{\circ} 2.714 .094\right)$.

\section{RESULTADOS}

Foram arrolados um total de 82 pacientes admitidos na UTI entre abril e setembro de 2018.

Os dados demográficos e de saúde da população do estudo são apresentados na Tabela 1 . No total, foram aferidas 11.676 glicemias capilares, com mediana de 91 por paciente. A mediana de idade foi 73 anos e o tempo de internação, 17 dias. A maior parte da coorte $(92,68 \%)$ fez uso de TN enteral, enquanto apenas $7,31 \%$ receberam nutrição parenteral. O tempo mediano de permanência com TN foi de 9 dias. Um total de 36,58\% dos pacientes tinham

Tabela 1 - Características demográficas, hospitalares e de saúde de pacientes hospitalizados.

\begin{tabular}{|c|c|}
\hline Variáveis & Valores $^{\mathrm{a}}$ \\
\hline Idade (anos) & $73(54-82)$ \\
\hline Número de glicemias aferidas & $91(36-185)$ \\
\hline Tempo de internação (dias) & $17(8-28)$ \\
\hline Tempo em terapia nutricional (dias) & $9(5-22)$ \\
\hline \multicolumn{2}{|l|}{ IMC } \\
\hline Adultos & $24,93 \mathrm{~kg} / \mathrm{m}^{2}\left(20,82-27,02 \mathrm{~kg} / \mathrm{m}^{2}\right)$ \\
\hline Idosos & $24,73 \mathrm{~kg} / \mathrm{m}^{2}\left(22,77-27,40 \mathrm{~kg} / \mathrm{m}^{2}\right)$ \\
\hline \multicolumn{2}{|l|}{ Sexo } \\
\hline Feminino & $51,21 \%(42)$ \\
\hline Masculino & $48,78 \%(40)$ \\
\hline \multicolumn{2}{|l|}{ Diabetes mellitus } \\
\hline Sim & $36,58 \%(30)$ \\
\hline Não & $63,41 \%(52)$ \\
\hline \multicolumn{2}{|l|}{ Desfecho } \\
\hline Alta & $57,31 \%(47)$ \\
\hline Óbito & $42,68 \%(35)$ \\
\hline \multicolumn{2}{|l|}{ Terapia nutricional } \\
\hline Enteral & $92,68 \%(76)$ \\
\hline Parenteral & $7,31 \%(6)$ \\
\hline \multicolumn{2}{|l|}{ Diagnóstico } \\
\hline Doenças cardíacas/vasculares & $21,95 \%(18)$ \\
\hline Doenças torácicas/respiratórias & $21,95 \%(18)$ \\
\hline Doenças do TGI & $6,09 \%(5)$ \\
\hline Doenças neurológicas & $25,60 \%(21)$ \\
\hline Traumas & $6,09 \%(5)$ \\
\hline Doenças neoplásicas & $7,31 \%(6)$ \\
\hline Outras & $10,97 \%(9)$ \\
\hline
\end{tabular}

$\mathrm{IMC}=$ índice de massa corporal; TGI = trato gastrintestinal.

Fonte: UTI de um hospital em Nova Lima/MG. Nota: ${ }^{R}$ Resultados expressos em mediana juntamente com o intervalo interquatilico correspondente e número absoluto e proporção correspondente. diagnóstico prévio de DM. Os principais diagnósticos de admissão foram doenças neurológicas, cardiovasculares e torácicas/respiratórias. Cerca de $42,7 \%$ dos pacientes da amostra foram a óbito.

Na Tabela 2, são apresentados os parâmetros glicêmicos entre os pacientes diabéticos e não-diabéticos. Como esperado, os pacientes com DM apresentaram valores significativamente maiores $(p \leq 0,01)$ para todos os parâmetros avaliados de variação glicêmica, $C V=28,43 \mathrm{mg} / \mathrm{dL}, \mathrm{DP}=$ $56,52 \mathrm{mg} / \mathrm{dL}$ e $\mathrm{MGL}=184,87 \mathrm{mg} / \mathrm{dL}$ em relação aos não-diabéticos $C V=22,41 \mathrm{mg} / \mathrm{dL}, \mathrm{DP}=29,46 \mathrm{mg} / \mathrm{dL}$ e MGL 143,90 mg/dL. O tempo de internação e o tempo em vigência de TN não foi estatisticamente diferente entre diabéticos e não-diabéticos ( $p=0,23$ e $p=0,13$, respectivamente).

Tabela 2 - Características demográficas, hospitalares e parâmetros glicêmicos entre pacientes diabéticos e não-diabéticos.

\begin{tabular}{|c|c|c|c|}
\hline Parâmetros ${ }^{a}$ & Diabéticos (30) & Não-diabéticos (52) & $p$ \\
\hline $\begin{array}{l}\text { Coeficiente de } \\
\text { variação } \\
\text { glicêmica }\end{array}$ & $\begin{array}{c}28,43 \\
(24,83-34,87)\end{array}$ & $\begin{array}{c}22,41 \\
(16,12-27,00)\end{array}$ & $\leq 0,01^{*}$ \\
\hline $\begin{array}{l}\text { Desvio padrão } \\
\text { glicêmico }\end{array}$ & $\begin{array}{c}56,52 \\
(44,95-69,82)\end{array}$ & $\begin{array}{c}29,76 \\
(20,06-43,70)\end{array}$ & $\leq 0,01^{*}$ \\
\hline $\begin{array}{l}\text { Média glicêmica } \\
(\mathrm{mg} / \mathrm{dL})\end{array}$ & $\begin{array}{c}184,87 \\
(163,51-216,31)\end{array}$ & $\begin{array}{c}143,90 \\
(128,48-164,68)\end{array}$ & $\leq 0,01^{*}$ \\
\hline $\begin{array}{l}\text { Tempo de terapia } \\
\text { nutricional (dias) }\end{array}$ & $\begin{array}{c}13 \\
(7-27)\end{array}$ & $\begin{array}{c}7 \\
(4-20)\end{array}$ & 0,13 \\
\hline $\begin{array}{l}\text { Tempo de } \\
\text { internação (dias) }\end{array}$ & $19(10-35)$ & $14(7-28)$ & 0,23 \\
\hline \multicolumn{4}{|l|}{ Idade } \\
\hline$\leq 60$ anos & $8(26,67 \%)$ & $18(34,62 \%)$ & 0,45 \\
\hline$>60$ anos & $22(73,33 \%)$ & $34(65,38 \%)$ & \\
\hline \multicolumn{4}{|l|}{ Sexo } \\
\hline Masculino & $17(56,67)$ & $23(44,23 \%)$ & 0,27 \\
\hline Feminino & $13(43,33)$ & $29(55,77 \%)$ & \\
\hline
\end{tabular}

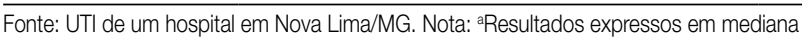
juntamente com o intervalo interquatílico correspondente e número absoluto e proporção correspondente. ${ }^{*} p<0,05$.

As características demográficas, hospitalares e parâmetros glicêmicos entre sobreviventes e não-sobreviventes são apresentados na Tabela 3. Os valores de CV, DP e MGL foram significativamente maiores $(p \leq 0,01)$ entre os pacientes que foram a óbito (CV=25,97 mg/dL, DP=50,45 mg/dL e $M G L=179,67$ $\mathrm{mg} / \mathrm{dL}$ ) em comparação aos sobreviventes ( $\mathrm{CV}=22,88 \mathrm{mg} / \mathrm{dL}$, $\mathrm{DP}=34,79 \mathrm{mg} / \mathrm{dL}$ e $M G L=149,43 \mathrm{mg} / \mathrm{dL}$ ). A mortalidade foi significativamente maior entre os pacientes que apresentavam mais de 60 anos $(p<0,04)$. Quando comparadas as populações de não-sobreviventes diabéticos e não-diabéticos, não foi observado significância $(p=0,27)$ entre os valores de CV $(28,52 \mathrm{mg} / \mathrm{dL} \times 25,53 \mathrm{mg} / \mathrm{dL})$, no entanto, o DP $(60,32 \mathrm{mg} /$ $\mathrm{dL} \times 41,30 \mathrm{mg} / \mathrm{dL})$ e a $M G L(211,51 \mathrm{mg} / \mathrm{dL} \times 165,28 \mathrm{mg} / \mathrm{dL})$ foram significativamente maiores entre os pacientes diabéticos $(p=0,03$ e $p \leq 0,01$, respectivamente). 


\begin{tabular}{|c|c|c|c|c|c|c|}
\hline Parâmetros $^{\mathrm{a}}$ & Sobreviventes (47) & Não-sobreviventes (35) & $p$ & $\begin{array}{c}\text { Óbitos } \\
\text { Não-diabéticos (20) }\end{array}$ & $\begin{array}{c}\text { Óbitos } \\
\text { Diabéticos (15) }\end{array}$ & $p$ \\
\hline $\begin{array}{l}\text { Coeficiente de } \\
\text { variação glicêmica }\end{array}$ & $22,88(16,34-28,34)$ & $25,97(22,36-33,26)$ & $\leq 0,01^{*}$ & $25,53(22,36-31,31)$ & $28,52(22,36-36,50)$ & 0,27 \\
\hline $\begin{array}{l}\text { Desvio padrão } \\
\text { glicêmico }\end{array}$ & $34,79(19,89-48,61)$ & $50,45(35,89-67,54)$ & $\leq 0,01^{*}$ & $41,30(31,50-58,16)$ & $60,32(46,95-73,08)$ & $0,03^{*}$ \\
\hline $\begin{array}{l}\text { Média glicêmica } \\
(\mathrm{mg} / \mathrm{dL})\end{array}$ & $149,43(128,10-169,60)$ & $179,67(157,56-211,51)$ & $\leq 0,01^{*}$ & $165,28(146,87-180,07)$ & $211,51(183,91-227,61)$ & $\leq 0,01^{*}$ \\
\hline $\begin{array}{l}\text { Tempo de terapia } \\
\text { nutricional }\end{array}$ & $12(5-23)$ & $7(4-22)$ & 0,49 & $7(4-12)$ & $14(5-30)$ & 0,15 \\
\hline $\begin{array}{l}\text { Tempo de } \\
\text { internação }\end{array}$ & $19(8-27)$ & $13(7-33)$ & 0,82 & $14(8-25)$ & $17(7-36)$ & 0,50 \\
\hline \multicolumn{7}{|l|}{ Idade } \\
\hline$\leq 60$ anos & $19(40,43 \%)$ & $7(20 \%)$ & $0,04^{*}$ & $5(25 \%)$ & $2(13,33)$ & 0,39 \\
\hline$>60$ anos & $28(59,57 \%)$ & $28(80 \%)$ & & 15 (75\%) & $13(86,67)$ & \\
\hline \multicolumn{7}{|l|}{ Sexo } \\
\hline Masculino & $22(46,81 \%)$ & $18(51,43 \%)$ & 0,67 & $9(45 \%)$ & $9(60 \%)$ & 0,38 \\
\hline Feminino & 25 (53,19\%) & $17(48,57 \%)$ & & 11 (55\%) & $6(40 \%)$ & \\
\hline
\end{tabular}

Fonte: UTI de um hospital em Nova Lima/ MG.

Nota: ${ }^{a}$ Resultados expressos em mediana juntamente com o intervalo interquatílico correspondente e número absoluto e proporção correspondente. * ${ }^{\star}<0,05$

A análise entre a mortalidade e fatores relacionados demonstrou que as variáveis CV, DP e MGL tiveram associação significativa (Tabela 4). No entanto, a partir do modelo de regressão logística multivariada, apenas o DP manteve relação com a mortalidade (Tabela 5).

Tabela 4 - Análise univariada da associação de características demográficas, hospitalares e parâmetros de variabilidade glicêmica com a mortalidade entre pacientes de uma unidade de terapia intensiva (UTI). Nova Lima, 2018.

\begin{tabular}{|c|c|c|}
\hline Parâmetros & Odds Ratio (IC) ${ }^{\mathrm{a}}$ & $p$ \\
\hline $\begin{array}{l}\text { Coeficiente de variação } \\
\text { glicêmica }\end{array}$ & $1,07(1,01-1,13)$ & $0,02^{*}$ \\
\hline Desvio padrão glicêmico & $1,04(1,01-1,06)$ & $\leq 0,01^{*}$ \\
\hline \multicolumn{3}{|l|}{ Média glicêmica } \\
\hline$<150$ & 1 & \\
\hline $150-200$ & $2,91(1,08-8,42)$ & $0,04^{*}$ \\
\hline$<200$ & $12,57(2,72-58,01)$ & $\leq 0,01^{*}$ \\
\hline $\begin{array}{l}\text { Diagnóstico confirmado de } \\
\text { diabetes mellitus }\end{array}$ & $1,60(0,64-3,96)$ & 0,31 \\
\hline Tempo de internação & $1,01(0,98-1,02)$ & 0,58 \\
\hline Idade & $1,02(1,01-1,05)$ & $0,02^{*}$ \\
\hline \multicolumn{3}{|l|}{ Sexo } \\
\hline Feminino & 1 & 0,67 \\
\hline Masculino & $0,83(0,34-1,99)$ & \\
\hline \multicolumn{3}{|l|}{ Tipo de terapia nutricional } \\
\hline Parenteral & 1 & 0,21 \\
\hline Enteral & $0,24(0,02-2,21)$ & \\
\hline
\end{tabular}

Fonte: UTI de um hospital em Nova Lima/MG

Nota: alC: Intervalo de confiança. ${ }^{*} p<0,05$
Tabela 5 - Modelo de regressão logística multivariada de associação com a mortalidade em pacientes de uma unidade de terapia intensiva (UTI). Nova Lima, 2018.

\begin{tabular}{lcc}
\hline Parâmetros & Odds Ratio (IC) & $\boldsymbol{p}$ \\
\hline Desvio Padrão & $1,03(1,01-1,06)$ & $\leq 0,01^{*}$ \\
\hline Fonte: UTI de um hospital em Nova Lima/MG. & \\
Nota: alC: Intervalo de confiança. ${ }^{*}<0,05$. Modelo final controlado pelas variáveis idade, média \\
glicêmica, coeficiente de variação glicêmica e tipo de terapia nutricional (enteral/parenteral).
\end{tabular}

\section{DISCUSSÃO}

A discussão ocorreu em torno do efeito da VG (avaliada pelo $D P, C V$ e $M G L$ ) na mortalidade em pacientes internados na UTI tratados com TN enteral e parenteral. Nesse estudo longitudinal retrospectivo, descobrimos que o DP é um preditor significativo e independente de mortalidade, corroborando com os achados de Egi et al. ${ }^{9}$, em um estudo observacional retrospectivo realizado em quatro hospitais australianos, e de Krinsley ${ }^{14}$, em uma revisão retrospectiva em um hospital universitário nos EUA.

Obtivemos diferenças clinicamente importantes entre pacientes portadores de DM e sem diabetes em relação a dois domínios de controle glicêmico (DP e MGL) e mortalidade. No entanto, a presença de DM não tem associação independente com a mortalidade e o aumento do CV, DP e MGL não foi associado ao aumento da mortalidade entre os pacientes com DM. Dentre os pacientes que foram a óbito, aqueles sem DM $(n=20)$ superam a quantidade dos portadores de DM que foram a óbito $(n=15)$. Resultados semelhantes foram 
encontrados por Krinsley et al. ${ }^{16}$, em um estudo retrospectivo realizado em 23 UTIs, em nove países, e que envolvia 44964 pacientes. Esses autores demonstraram que o diagnóstico de DM correlacionou-se com a diminuição do risco de morte e que o aumento do VG foi significativamente associado a um aumento na mortalidade em pacientes não-diabéticos, mas não em pacientes com $\mathrm{DM}^{16}$. Da mesma forma, Farrokhi et al. ${ }^{17}$, ao estudarem uma coorte de 276 pacientes médicos e cirúrgicos com TN parenteral, relataram que a associação entre VG e mortalidade foi significativa apenas em pacientes não-diabéticos.

Os mecanismos pelos quais o DM pode ser um fator protetor em relação à mortalidade, em pacientes gravemente doentes, são desconhecidos, mas podem estar relacionados à hiperglicemia crônica, que resulta em condicionamento celular que é protetor contra os danos causados pela hiperglicemia aguda durante a doença crítica ${ }^{18-20}$.

Outras hipóteses que não estão relacionadas ao controle glicêmico podem ajudar a explicar o paradoxo do diabetes, como maior reserva nutricional em pacientes obesos com DM tipo 2, adaptação ao estresse oxidativo prévio e alguns medicamentos usados por esses pacientes podem minimizar o curso de doenças críticas ${ }^{21}$.

Devemos ressaltar que, no presente estudo, a coorte era composta exclusivamente por pacientes que fizeram o uso de $\mathrm{TN}$ e, apesar dessa variável não ter apresentado significância, devemos considerá-la, pois na análise univariada a TN apresentou $p=0,21$. Estudos demonstraram que a $T N$, seja ela enteral ou parenteral, pode agravar as oscilações de glicemia, principalmente se o início é repentino e/ou as pausas frequentes, sendo as pausas comuns em pacientes críticos, devido à instabilidade hemodinâmica, à intolerância à $\mathrm{TN}$ e à realização de procedimentos e exames ${ }^{15,22}$. $\mathrm{O}$ que, por sua vez, pode alterar a VG e, consequentemente, influenciar na mortalidade.

As limitações do nosso estudo são o fato de ser unicêntrico e de natureza retrospectiva, apresentando todas as limitações inerentes a tais estudos. Os pontos fortes incluem que os dados foram coletados prospectivamente, por apenas um pesquisador e a administração de insulina por via intravenosa foi realizada conforme protocolo pré-definido.

\section{CONCLUSÃO}

O estudo demonstrou que a VG medida pelo DP é um preditor independentemente de mortalidade na UTI em uma coorte de pacientes heterogêneos em uso de TN.

Nenhuma diretriz propôs um padrão-ouro para medir a VG, no entanto, descobrimos que a $M G L$ e $C V$ não tem associação com a mortalidade, sendo o DP o único dos três domínios que apresentou significância estatística. Esses achados precisam ser confirmados por ensaios multicêntricos maiores. Se a TN melhora ou não os resultados, também é necessário ser elucidado em estudos futuros. No entanto, recomendamos que protocolos institucionalizados sejam implementados para o manejo da hiperglicemia em pacientes críticos, enfatizando tanto o bom controle glicêmico quanto a minimização da VG.

\section{AGRADECIMENTOS}

À nutricionista Juliana Souza, pelo auxílio estatístico, e à equipe do hospital onde a pesquisa foi realizada.

\section{REFERÊNCIAS}

1. Van den Berghe G, Wouters P, Weekers F, Verwaest C, Bruyninckx F, Schetz $\mathrm{M}$, et al. Intensive insulin therapy in critically ill patients. N Engl J Med. 2001;345(19):1359-67.

2. Preiser JC, Devos P, Ruiz-Santana S, Mélor C, Annane D, Groeneveld J, et al. A prospective randomised multi-centre controlled trial on tight glucose control by intensive insulin therapy in adult intensive care units: the Glucontrol study. Intensive Care Med. 2009;35(10):1738-48.

3. Van den Berghe G, Wilmer A, Hermans G, Meersseman W, Wouters PJ, Milants I, et al. Intensive insulin therapy in the medical ICU. N Engl J Med. 2006;354(5):449-61.

4. NICE-SUGAR Study Investigators for the Australian and New Zealand Intensive Care Society Clinical Trials Group and the Canadian Critical Care Trials Group. Finfer S, Chittock D, Li Y, Foster D, Dhingra V, Bellomo R, et al. Intensive versus conventional glucose control in critically ill patients with traumatic brain injury: long-term follow-up of a subgroup of patients from the NICE-SUGAR study. Intensive Care Med. 2015;41(6):1037-47.

5. BrunkhorstFM, EngelC, Bloss F, Meier-HellmannA, RagallerM, Weiler N, et al. Intensive insulin therapy and pentastarch resuscitation in severe sepsis. N Engl J Med. 2008;358(2):125-39.

6. Todi S, Bhattacharya M. Glycemic variability and outcome in critically ill. Indian J Crit Care Med. 2014;18(5):285-90.

7. Krinsley JS. Glycemic variability and mortality in critically ill patients: the impact of diabetes. J Diabetes Sci Tchnol. 2009;3(6):1292-301.

8. Brunner R, Adelsmayr G, Herkner H, Madl C, Holzinger U. Glycemic variability and glucose complexity in critically ill patients: a retrospective analysis of continuous glucose monitoring data. Crit Care. 2012;16(5):R175.

9. Egi M, Bellomo R, Stachowski E, French CJ, Hart G. Variability of blood glucose concentration and short-term mortality in critically ill patients. Anesthesiology. 2006;105(2):244-52.

10. Hermanides J, Vriesendorp TM, Bosman RJ, Zandstra DF, Hoekstra JB, Devries JH. Glucose variability is associated with intensive care unit mortality. Crit Care Med. 2010;38(3):838-42.

11. Ali NA, O’Brien JM Jr, Dungan K, Phillips G, Marsh CB, Lemeshow S, et al. Glucose variability and mortality in patients with sepsis. Crit Care Med. 2008;36(8):2316-21.

12. Dossett LA, Cao H, Mowery NT, Dortch MJ, Morris JM Jr, May AK. Blood glucose variability is associated with mortality in the surgical intensive care unit. Am Surg. 2008;74(8):679-85.

13. Meynaar IA, Eslami S, Abu-Hanna A, Vam der Voort P, Lange DW, Keizer N. Blood glucose amplitude variability as predictor for mortality in surgical and medical intensive care unit patients: a multicenter cohort study. J Crit Care. 2012;27(2):119-24. 
14. Krinsley JS. Glycemic variability: a strong independent predictor of mortality in critically ill patients. Crit Care Med. 2008;36(11):3008-13.

15. Clain J, Ramar K, Surani SR. Glucose control in critical care. World J Diabetes. 2015;6(9):1082-91.

16. Krinsley JS, Egi M, Kiss A, Devendra AN, Schuetz P, Maurer PM, et al. Diabetic status and the relation of the three domains of glycemic control to mortality in critically ill patients: an international multicenter cohort study. Crit Care. 2013;17(2):R37.

17. Farrokhi F, Chandra P, Smiley D, Pasquel FJ, Peng L, Newton $\mathrm{CA}$, et al. Glucose variability is an independent predictor or mortality in hospitalized patients treated with total parenteral nutrition. Endrocr Pract. 2014;20(1):41-5.

18. Siegelaar SE, Holleman F, Hoekstra JB, DeVries JH. Glucose variability; does is matter? Endocr Rev. 2010;31(2):171-82.
19. Schisano B, Tripathi G, McGee K, McTerman PG, Ceriello A. Glucose oscillations, more than constant high glucose, induce p53 activation and a metabolic memory in human endothelial cells. Diabetologia. 2011;54(5):1219-26.

20. Klip A, Tsakiridis T, Marette A, Ortiz PA. Regulation of expression of glucose transporters by glucose: a review of studies in vivo and in cell cultures. FASEB J. 1994;8(1):43-53.

21. Krinsley JS, Fisher M. The diabetes paradox: diabetes is not independently associated with mortality in critically ill patients. Hosp Pract. 2012;40(2):31-5.

22. McMahon MM, Nystrom E, Braunschweiq C, Miles J, Compher C, American Society for Parenteral and Enteral Nutrition (A.S.P.E.N.) Board of Directors, American Society for Parenteral and Enteral Nutrition. Clinical guidelines: nutrition support of adult patients with hyperglycemia. JPEN J Parenter Enteral Nutr. 2013;37(1):23-36.

Local de realização do estudo: Hospital Vila da Serra, Nova Lima, MG, Brasil.

Conflito de interesse: Os autores declaram não haver.

Foi apresentado como trabalho de destaque no XXIII Congresso Brasileiro de Nutrição Parenteral e Enteral, entre 20 a 23 de outubro de 2019, em Foz do Iguaçu-PR. 